\title{
Power Quality Disturbance Classification Method Based on Wavelet Transform and SVM Multi-class Algorithms
}

\author{
Xiao Fei \\ Southwest Jiaotong University, School of Electrical Engineering, ChengDu, China \\ Email: 776526963@qq.com
}

Received March, 2013

\begin{abstract}
The accurate identification and classification of various power quality disturbances are keys to ensuring high-quality electrical energy. In this study, the statistical characteristics of the disturbance signal of wavelet transform coefficients and wavelet transform energy distribution constitute feature vectors. These vectors are then trained and tested using SVM multi-class algorithms. Experimental results demonstrate that the SVM multi-class algorithms, which use the Gaussian radial basis function, exponential radial basis function, and hyperbolic tangent function as basis functions, are suitable methods for power quality disturbance classification.
\end{abstract}

Keywords: Power Quality; Disturbance Classification; Wavelet Transform; SVM Multi-Class Algorithms

\section{Introduction}

Increasingly superior electrical power supply has become necessary with the development and extensive application of electricity and electronics technology. However, all types of non-linear impact loads worsen electrical energy pollution. Given this backdrop, researchers have directed considerable attention to power quality disturbance classification because of its ability to determine the cause of energy disturbance and improve power quality. This approach is currently an important area of research on power systems.

The commonly used methods for extracting power disturbance features are wavelet transforms [1], Fourier transforms [2], and S transforms [3]. These techniques share certain attributes and can effectively extract energy characteristics. Nevertheless, the accuracy of these classification methods is tremendously affected by environmental noise. Other available methods include neural network classification [4], support vector machine [5], and particle swarm optimization [6], which is typically used to classify disturbance signals. These methods are similar in that they require effective training samples, as well as present high classification accuracy, high computational complexity, and weak classification for multiclass samples.

In this paper, we use the wavelet analysis method to extract the effective feature vectors of power quality disturbances, and regard these vectors as SVM training samples. We take advantage of multi-class SVM in classifying different power disturbance scenarios, such as voltage sag, voltage swell, voltage interruption, pulse transient, and harmonic classification. Multi-class SVM presents higher classification accuracy and efficiency in power systems than do other classifiers.

\section{Feature Vectors of Extraction Based on Wavelet Transform}

The wavelet transform concept was originally proposed by French geophysicist J. Morlet in 1984. Theoretical physicist A. Grossman established the theoretical system of wavelet transform on the basis of the theory of translation and scale invariance. French mathematician Y. Meyer constructed the first wavelet.

The Fourier transform is a useful tool for analyzing the frequency components of a signal. However, the window length used in this operation limits frequency resolutions. Wavelet transforms are based on small wavelets with limited durations; thus, they present higher frequency resolutions at low frequencies and low time resolutions. They also exhibit higher time resolutions and lower frequency resolutions at high frequencies. With these properties, wavelet transforms are adaptive to signal analysis.

Wavelet transform involves the displacement of basic wavelet functions $\psi(t)$. Then, the inner products of signals $x(t)$ and $\psi(t)$ are calculated under different scales thus:

$$
W T_{x}(a, \tau)=\frac{1}{\sqrt{a}} \int_{-\infty}^{+\infty} x(t) \psi^{*}\left(\frac{t-\tau}{a}\right) a>0 ;
$$

where $\tau$ is the translating parameter that indicates the 
region of interest; $a$ denotes the scaling parameter or scale, and measures the degree of compression. In the frequency domain, this function is expressed as

$$
W T_{x}(a, \tau)=\frac{\sqrt{a}}{2 \pi} \int_{-\infty}^{+\infty} x(\omega) \psi^{*}(a \omega) e^{j \omega \tau} d \omega,
$$

where $x(\omega)$ is the Fourier transform of $x(t) ; \psi(\omega)$ is the Fourier transform of $\psi(t)$.

Selecting an appropriate wavelet converts $\psi(t)$ in the time domain into a finite support and turns $\psi(\omega)$ in the frequency domain into a relatively concentrated variable. Implementing wavelet transform in the time and frequency domains also characterizes local signal features. The energy distribution of various power disturbance signals differs at various frequency bands. Thus, we can incorporate different energy distributions in different frequency bands as bases for distinguishing power disturbances.

On the basis of references [7, 8], we choose sym4 as the mother wavelet, and decompose power disturbance signals into 11 layers. A total of 23 characteristic values constitute a feature vector. $V_{1} \sim V_{11}$ represents the quadratic sum of the eleventh to the first layers of coefficients. $V_{12} \sim V_{23}$ are calculated as follows [9]:

$$
\begin{aligned}
& V_{12}=\frac{s t d\left|D_{11}\right|}{\text { mean }\left|D_{11}\right|}, \quad V_{13}=\frac{s t d\left|D_{10}\right|}{\text { mean }\left|D_{10}\right|}, \\
& V_{14}=\frac{s t d\left|D_{11}\right|}{\text { mean }\left|D_{11}\right|}, \quad V_{15}=\frac{s t d\left|D_{8}\right|}{\text { mean }\left|D_{8}\right|}, \\
& V_{16}=\frac{s t d\left|D_{7}\right|}{\text { mean }\left|D_{7}\right|}, \quad V_{17}=\frac{s t d\left|D_{6}\right|}{\text { mean }\left|D_{6}\right|}, \\
& V_{18}=\frac{s t d\left|D_{5}\right|}{\text { mean }\left|D_{5}\right|}, \quad V_{19}=\frac{\text { std }\left|D_{4}\right|}{\text { mean }\left|D_{4}\right|}, \\
& V_{20}=\frac{\operatorname{std}\left|D_{11,10,9}\right|}{\text { mean }\left|D_{11,10,9}\right|}, \quad V_{21}=\frac{s t d\left|D_{8,7}\right|}{\text { mean }\left|D_{8,7}\right|}, \\
& V_{22}=\frac{\operatorname{std}\left|D_{6,5,4}\right|}{\text { mean }\left|D_{6,5,4}\right|}, \quad V_{23}=\frac{\text { std }\left|D_{3,2,1}\right|}{\text { mean }\left|D_{3,2,1}\right|},
\end{aligned}
$$

where std $\left|D_{11}\right|$ is the standard deviation of the eleventh layer of decomposition coefficients, std $\left|D_{11,10,9}\right|$ denotes the standard deviation of the ninth to eleventh layers of decomposition coefficients, and mean $\left|D_{11}\right|$ represents the average of the absolute value of decomposition coefficients.

\section{Multi-class SVM Classification Model}

The commonly used multi-class SVM classification algorithm is 1-to-1 (1-vs-SVM). When a classification problem is highly complicated, however, training time and computational complexity significantly increase. To illustrate, let us consider $k$ types of samples that need to be classified. To solve this problem, we construct a $k(k-1) / 2$ hyper-plane.

To reduce computational complexity, researchers created another classification algorithm, 1-VS-allSVM, this involves hyper-plane classification that distinguishes between one class of samples and the rest of several class samples. Only the $k(k>2)$ hyper-plane can solve the previous problem-- $k$ types of samples that need to be classified. This method is an extension of two types of SVM. The prediction accuracy of the classifier is imperfect because of the huge difference between the number of a single class of samples and the number of the rest of the class samples.

In this paper, we use the BT-SVM method to improve the accuracy and efficiency of the classifier. $k(k>2)$ types of samples require classification. First, we construct SVM1 by assigning the $1^{\text {st }}$ sample type as a positive sample and the $2^{\text {nd }}, 3^{\text {rd }} \ldots \mathrm{k}^{\text {th }}$ types as negative samples. We then construct SVM 2 by denoting the $2^{\text {nd }}$ sample type as a positive sample and the $3^{\text {rd }}, 4^{\text {th }} \ldots \mathrm{k}^{\text {th }}$ types as negative samples. According to the previous method, we construct subsequent classifiers SVM $3 \cdots$ SVM $(k-1)$. The number of negative samples gradually decreases; training time also decreases. We choose BT-SVM [10] to classify different scenarios of power quality disturbance. The algorithm is implemented as follows:

1) Divide training sample $C_{i}(i=1,2, \ldots, k)$ into subsets $T$ and $F$, and regard $T, F$ as positive and negative samples, respectively. These samples consist of a classification function $f_{i}(x)\left(i=1,2, \ldots, 2^{N}\right)$.

2) Take $f_{i}(x)$ as the root node for constructing a binary tree.

3) Repeat steps (1) and (2), then use $\mathrm{T}$ as the training data for the left subtree and $\mathrm{F}$ to generate a classification function for the right subtree. $\mathrm{T}$ is also used to construct the classification function.

4) Repeat step (3) until training sample $C_{i}(i=1,2$, $\ldots, k)$ is converted into a group of child nodes;

5) Input testing sample $x_{i} \in c_{i}$ to the corresponding binary tree $f_{i}(x)$.

6) If all testing samples $x_{i} \in c_{i}$ belong to the ith sample type, then the classification is completed. The same applies when all testing samples $x_{i} \in c_{i}$ are required to traverse from the root node to pass through all the nodes until the category to which the samples belong are identified.

We use different support vector machines to match different scenarios of power quality disturbance. Thus, every support vector machine can solve a particular classification problem and improve accuracy by using training samples. 


\section{Simulation and Analysis}

\subsection{Types of Power Quality Disturbances}

In the simulation experiment, we construct six types of power disturbance models: voltage sag, voltage swell, voltage interruption, oscillating transient, harmonic, and flicker. The mathematical models are as follows:

1) Normal signal model

$$
z(t)=A \sin \omega t
$$

where $A$ is the amplitude of a signal, $\omega$ denotes the frequency of the signal, and $t$ represents time.

2) Voltage interruption model

$$
z(t)=A\left[1-\alpha\left(u\left(t-t_{1}\right)-u\left(t-t_{2}\right)\right)\right] \sin \omega t
$$

where $\alpha$ is the amplitude of an additional oscillation signal and $0.9 \leq \alpha \leq 0.99$; here, $T$ is the period of the signal. We assume that $T \leq t_{2}-t_{1} \leq 8 T$, the start time of a disturbance is $t_{1}$, and the completion time of disturbance is $t_{2} \cdot u(t)$ is a step function.

3) Voltage sag model

$$
z(t)=A\left[1-\alpha\left(u\left(t-t_{1}\right)-u\left(t-t_{2}\right)\right)\right] \sin \omega t
$$

where $\alpha$ is the amplitude of the additional oscillation signal and $0.1 \leq \alpha \leq 0.9$; here, $T \leq t_{2}-t_{1} \leq 8 T$.

4) Voltage swell model

$$
z(t)=A\left[1+\alpha\left(u\left(t-t_{1}\right)-u\left(t-t_{2}\right)\right)\right] \sin \omega t
$$

where $0.1 \leq \alpha \leq 0.5$ and $T \leq t_{2}-t_{1} \leq 8 T$.

5) Harmonic model

$$
\begin{aligned}
z(t)= & A\left(\sin (\omega t)+\alpha_{3} \sin (3 \omega t)+\alpha_{5} \sin (5 \omega t)\right. \\
& \left.+\alpha_{7} \sin (7 \omega t)+\alpha_{11} \sin (11 \omega t)\right)
\end{aligned}
$$

where $0.05 \leq \alpha_{i} \leq 0.15$.

6) Oscillating transient model

$$
\begin{aligned}
z(t)= & \sin (\omega t)+\alpha_{\text {osc }} \exp \left(-\left(t-t_{b}\right) / \tau_{\text {osc }}\right) \\
& * \sin \left(\omega_{\text {nosc }}\left(t-t_{b}\right)\right)
\end{aligned}
$$

where $\tau_{\text {osc }}$ is the oscillation constant, $\omega_{\text {nosc }}$ denotes the oscillation frequency, and $\alpha_{\text {osc }}$ is the amplitude of the additional oscillation signal. $\tau_{\text {osc }} \in(0.008,0.04) s$, $\omega_{\text {nosc }} \in(100,400) \mathrm{Hz}$, and $0.1<\alpha_{\text {osc }}<0.6$.

7) Flicker model

$$
z(t)=A(1+\alpha \sin \beta \omega t) \sin \omega t
$$

where $0.1 \leq \alpha_{i} \leq 0.2 \quad 0.1 \leq \beta \leq 0.2$.

We assume that $A=220, f=50, \omega=2 * 3.14 * 50$, and the rest of the parameters are constrained by their own models. The six types of power transient disturbances are illustrated in Figure 1.

\subsection{Classification of Power Quality Disturbances}

We construct six types of mathematical models to simulate electric power disturbances, namely, voltage inter- ruption, voltage sag, voltage swell, harmonics, oscillating transient, and flicker. We also simulate 600 sets of samples with every type of disturbance scenario. We use a multi-class SVM algorithm to classify the samples. The four different types of kernel functions are the Gaussian radial basis function (GRBF), exponential radial basis function (ERBF), hyperbolic tangent function (HTF), and polynomial function (PF). These are used in SVM algorithms. To obtain an accurate, credible result, we carry

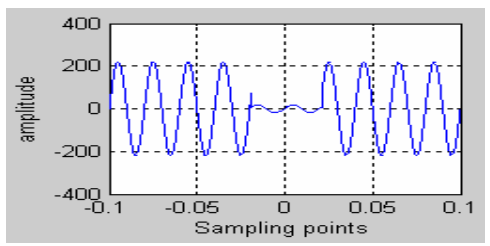

(a) voltage interruption

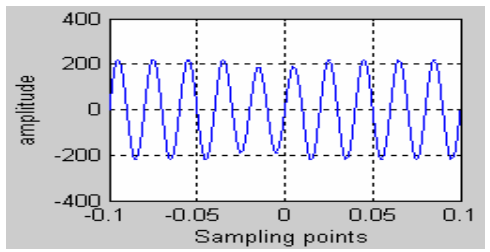

(b) voltage sag

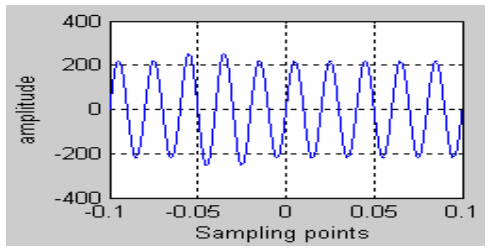

(c) voltage swell

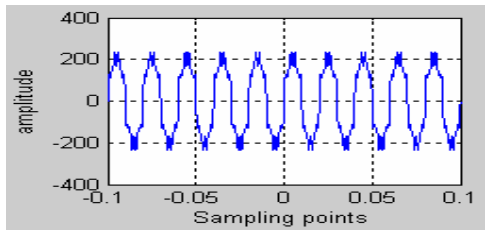

(d) harmonics

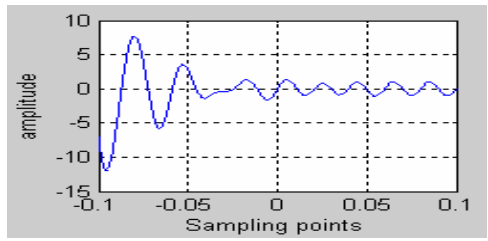

(e) oscillating transient

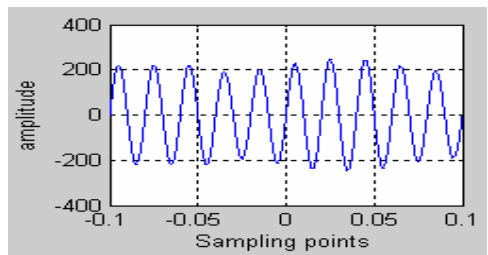

(f) flicker

Figure 1. Oscillogram of various disturbance signals. 
out calculations 10 times for every kernel function and adopt 150 or 160 sets of samples each time. The classification results are shown in Tables 1-6.

Table 1. Classification results for voltage interruption.

\begin{tabular}{cccccc}
\hline & GRBF & ERBF & HTF & PF 1 & PF2 \\
\hline & K=2 & K=3 & K=4 & K=11 & K=12 \\
1 & 150 & 150 & 150 & 73 & 134 \\
2 & 150 & 150 & 150 & 135 & 150 \\
3 & 150 & 150 & 150 & 77 & 137 \\
4 & 150 & 150 & 150 & 143 & 74 \\
5 & 150 & 150 & 150 & 67 & 85 \\
6 & 150 & 150 & 150 & 114 & 109 \\
7 & 150 & 150 & 150 & 75 & 145 \\
8 & 150 & 150 & 150 & 100 & 73 \\
9 & 150 & 150 & 150 & 107 & 94 \\
10 & 150 & 150 & 150 & 71 & 145 \\
& $100 \%$ & $100 \%$ & $100 \%$ & $64.1 \%$ & $76.4 \%$ \\
\hline
\end{tabular}

Table 2. Classification results for voltage sag.

\begin{tabular}{cccccc}
\hline & GRBF & ERBF & HTF & PF 1 & PF2 \\
\hline & K=2 & K=3 & K=4 & K=11 & K=12 \\
1 & 160 & 160 & 160 & 159 & 129 \\
2 & 160 & 160 & 160 & 96 & 78 \\
3 & 160 & 160 & 160 & 59 & 119 \\
4 & 160 & 160 & 160 & 70 & 95 \\
5 & 160 & 160 & 160 & 156 & 130 \\
6 & 160 & 160 & 160 & 83 & 104 \\
7 & 160 & 160 & 160 & 128 & 100 \\
8 & 160 & 160 & 160 & 113 & 79 \\
9 & 160 & 160 & 160 & 157 & 127 \\
10 & 160 & 160 & 160 & 81 & 69 \\
& $100 \%$ & $100 \%$ & $100 \%$ & $68.9 \%$ & $64.4 \%$ \\
\hline
\end{tabular}

Table 3. Classification results for voltage swell.

\begin{tabular}{cccccc}
\hline & GRBF & ERBF & HTF & PF 1 & PF2 \\
\hline & K=2 & K=3 & K=4 & K=11 & K=12 \\
1 & 150 & 150 & 150 & 137 & 81 \\
2 & 150 & 150 & 150 & 87 & 150 \\
3 & 150 & 150 & 150 & 132 & 131 \\
4 & 150 & 150 & 150 & 78 & 78 \\
5 & 150 & 150 & 150 & 115 & 82 \\
6 & 150 & 150 & 150 & 96 & 76 \\
7 & 150 & 150 & 150 & 84 & 40 \\
8 & 150 & 150 & 150 & 111 & 45 \\
9 & 150 & 150 & 150 & 140 & 148 \\
10 & 150 & 150 & 150 & 121 & 105 \\
& $100 \%$ & $100 \%$ & $100 \%$ & $73.4 \%$ & $62.4 \%$ \\
\hline
\end{tabular}

Table 4. Classification results for harmonics.

\begin{tabular}{cccccc}
\hline & GRBF & ERBF & HTF & PF 1 & PF2 \\
\hline & K=2 & K=3 & K=4 & K=11 & K=12 \\
1 & 160 & 160 & 160 & 149 & 41 \\
2 & 160 & 160 & 160 & 132 & 125 \\
3 & 160 & 160 & 160 & 131 & 56 \\
4 & 160 & 160 & 160 & 120 & 91 \\
5 & 160 & 160 & 160 & 67 & 74 \\
6 & 160 & 160 & 160 & 75 & 93 \\
7 & 160 & 160 & 160 & 87 & 49 \\
8 & 160 & 160 & 160 & 153 & 148 \\
9 & 160 & 160 & 160 & 137 & 160 \\
10 & 160 & 160 & 160 & 56 & 112 \\
& $100 \%$ & $100 \%$ & $100 \%$ & $69.2 \%$ & $69.3 \%$ \\
\hline
\end{tabular}

Table 5. Classification results for $f$ oscillation transient.

\begin{tabular}{cccccc}
\hline & GRBF & ERBF & HTF & PF 1 & PF2 \\
\hline & K=2 & K=3 & K=4 & K=11 & K=12 \\
1 & 160 & 160 & 160 & 110 & 41 \\
2 & 160 & 160 & 160 & 160 & 66 \\
3 & 160 & 160 & 160 & 135 & 43 \\
4 & 160 & 160 & 160 & 78 & 160 \\
5 & 160 & 160 & 160 & 119 & 88 \\
6 & 160 & 160 & 160 & 160 & 158 \\
7 & 160 & 160 & 160 & 160 & 129 \\
8 & 160 & 160 & 160 & 108 & 65 \\
9 & 160 & 160 & 160 & 136 & 149 \\
10 & 160 & 160 & 160 & 40 & 109 \\
& $100 \%$ & $100 \%$ & $100 \%$ & $75.4 \%$ & $63.0 \%$ \\
\hline
\end{tabular}

Table 6. Classification results for oscillation transient.

\begin{tabular}{cccccc}
\hline & GRBF & ERBF & HTF & PF 1 & PF2 \\
\hline & K=2 & K=3 & K=4 & K=11 & K=12 \\
1 & 160 & 160 & 160 & 136 & 85 \\
2 & 160 & 160 & 160 & 148 & 53 \\
3 & 160 & 160 & 160 & 97 & 137 \\
4 & 160 & 160 & 160 & 95 & 70 \\
5 & 160 & 160 & 160 & 75 & 157 \\
6 & 160 & 160 & 160 & 119 & 113 \\
7 & 160 & 160 & 160 & 80 & 81 \\
8 & 160 & 160 & 160 & 140 & 88 \\
9 & 160 & 160 & 160 & 129 & 160 \\
10 & 160 & 160 & 160 & 87 & 72 \\
& $100 \%$ & $100 \%$ & $100 \%$ & $69.1 \%$ & $63.5 \%$ \\
\hline
\end{tabular}


The multi-class SVM classifier with GRBF, ERBF, and HTF are suitable for classifying six types of electric power disturbances. By contrast, PF should not be used to solve such disturbances. As indicated by the results, the choice of kernel function considerably influences the classification results of multi-class SVM.

\section{Conclusions}

Using wavelet transforms to decompose electric power disturbance signals into 11 layers and extract each layer's detail coefficients as feature vectors can improve the accuracy of classification results. In classifying different types of electric power disturbance signals, the suitable kernel functions for multi-class SVM algorithms are GRBF, ERBF, and HTF. Multi-class SVM classification combined with wavelet transform can be an efficient method for differentiating power quality disturbance signals.

\section{REFERENCES}

[1] A. M. Gaouda, M. M. A. Salama and M. R. Sultan, "Power Quality Detection and Classification Using Wavelets Multi-resolution Signal Decomposition,” IEEE Trans on Power Delivery, Vol. 14, No. 4, 1999, pp. 1469-1476.doi: 10.1109/61.796242

[2] Y. Liao and J. B. Lee, “A Fuzzy-expert System for Clas sifying Power Quality Disturbances,” International Journal of Electrical Power and Energy Systems, Vol. 26, No. 3, 2004, pp.199-205. doi: 10.1016/j.ijepes. 2003.10.012

[3] J. L. Yi and J. C. Peng, "Classification of Short-time
Power Quality Disturbance Signals Based on Generalized S-transform,” Power System Technology, Vol. 33, No. 5, 2009, pp. 22-27.

[4] Y. F. Ren, H. S. Li and H. T. Hu, "Parallel Power Quality Controller Based on Multilayer Feedforward Neural Network,” Transactions of China Electrical Society, Vol. 22, No. 8, 2007, pp. 108-113.

[5] G. Y. Li, H. L. Wang and M. Zhou, "Short-time Power Quality Disturbances Identification Based on Improved Wavelet Energy Entropy and SVM," Transactions of China Electrical Society, Vol. 24, No. 4, 2009, pp. 161-167.

[6] G. H. Yang and B. Y. Wen, "Identification of Power Quality Disturbance Based on QPSO-ANN," Journal of China Motor Engineering, Vol. 28, No. 10, 2008, pp. 123-129.

[7] P. Janik and T. Lobos, "Automated Classification of Power-quality Disturbances Using SVM and RBF Networks," IEEE Transaction on Power Delivery, Vol. 21, No. 3, 2006, pp. 1663-1669.doi: 10.1109/TPWRD. 2006.874114

[8] J. G. Yao, Z. F. Guo and J. P. Chen, “A New Approach to Recognize Power Quality Disturbances Based on Wavelet Transform and BP Neural Network," Power System Technology, Vol. 36, No. 5, 2012, pp. 139-144.

[9] N. Hamzah, F. H. Anuwar and Z. Zakaria, "Classification of Transient in Power System Using Support Vector Machine," 5th international colloquium on signal processing \& its applications, Kuala Lumpur Malaysia: IEEE, 2009, pp. 418-422.

[10] Z. Wang, C. L. Wang and L. liu, "Improvement on Bintree Multi-class Categorization Algorithm Based on SVM,” Journal of Wuhan Institute of Technology, Vol. 32, No. 7, 2010, pp. 89-93. 